\title{
THE PSYCHOSOCIAL CHALLENGES FACING MULTI DRUG RESISTANCE TUBERCULOSIS PATIENTS: A QUALITATIVE STUDY
}

\author{
Thiruvalluvan $\mathrm{E}^{1}$, Thomas $\mathrm{B}^{2}$, Suresh $\mathrm{C}^{2}$, Sellappan $\mathrm{S}^{2}$, Muniyandi $\mathbf{M}^{2}$, Watson $\mathrm{B}^{3}$ \\ 'Department of Socio-behavioural Research, National Institute for Research in Tuberculosis (ICMR), Madurai \\ ${ }^{2}$ Department of Socio-behavioural Research, National Institute for Research in Tuberculosis (ICMR), Chennai \\ ${ }^{3}$ Department of Statistics, National Institute for Research in Tuberculosis (ICMR), Chennai
}

\begin{abstract}
Background: The treatment for MDR-TB characterized by rigorous treatment regimen for long duration, higher incidence of adverse side effects, lower cure rate, and high treatment costs. This could lead to number of psychosocial problems that influence treatment adherence. MDR-TB patients registered under DOTS Plus programme during the period of 2013-2014 in Chennai and Madurai districts, of Tamilnadu were included for this study.
\end{abstract}

Objective: To understand the psychosocial issues facing MDR-TB patients, who are diagnosed and registered for treatment under DOTS plus programme.

Methodology: This study used Focus Group Discussions with people with MDR-TB. Focus Group Discussions were focused on physical, psychological, social and economical challenges which MDR-TB patients faced during their treatment.

Results: Most of the study participants did not disclose their TB status, even to their family members. The majority of patients were not aware of the diagnosis of MDR-TB and long duration of treatment. Stigma from family, community and health providers has been experienced by the majority of patients. Patients and their families were afraid of losing economic stability which was already precarious owing to the disease. This fear has often generated a great deal of stress.

Conclusion: Study finding indicates that there is a significant psychological, social, and financial impact of MDR-TB that has a direct impact on quality of life of MDR-TB patients and their families. There is a need for psychosocial intervention model (strategies) for MDR-TB patients and their caregivers to mitigate the negative effects.

Key words: Tuberculosis, Multi Drug Resistant Tuberculosis (MDR-TB), Psychosocial Issues, Stigma, Economic Impact

\section{INTRODUCTION}

Multidrug resistant tuberculosis (MDR-TB) is defined as resistance to, at least, rifampicin and isoniazid, the two most powerful first-line anti-TB drugs. Globally, the proportion ${ }^{1}$ of new cases with multidrug-resistant TB (MDR-TB) was 3.5\% in the year 2013.Drug resistance surveillance data show

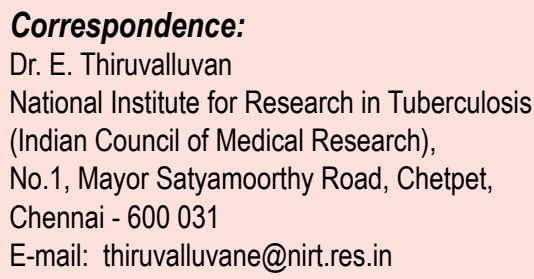

that an estimated 480,000 people developed MDRTB in 2013 of which 210,000 people died. Treatment outcomes of MDR-TB documented a success rate of $48 \%$, death $16 \%$, interrupted treatment $24 \%$ and failure $12 \% .{ }^{2}$ In India, the available information from the several drug resistance surveillance studies suggests that the prevalence of MDR-TB is $2-3 \%$ among new cases and $12-17 \%$ among re-infection cases. ${ }^{3}$ It was estimated that out of 73,000 MDR-TB patients living in India, only 1,660 cases were notified and 1,136 cases $(1.6 \%)$ were put on treatment. ${ }^{4}$

The treatment for MDR-TB characterised by rigorous treatment regimen for long duration, higher incidence of adverse side effects, lower cure rate, and high treatment costs. ${ }^{5}$ This could 
lead to number of psychosocial problems that influence treatment adherence. Recognising these problems associated with MDR-TB, 'DOTS Plus' guideline developed by the Ministry of Health and Family Welfare, Government of India recommends for an integrated approach to treat this disease. DOTS Plus strategy, consists of a multidisciplinary team of providers which includes physicians, nurses, social workers, community health workers and volunteers who provide a range of services for the MDR-TB patients including psycho social and community support. ${ }^{6}$ While several studies in India and other countries have investigated psychosocial problems associated with TB patients, still there is a dearth of studies among MDR-TB patients in the same context. The present study was undertaken to explore and understand the psychosocial issues that negatively impact the treatment adherence among MDR-TB patients, who have been diagnosed and registered for treatment under RNTCP.

\section{METHODOLOGY}

This qualitative study utilized FGDs and interviews covering 83 (68 MDR-TB patients and $15 \mathrm{HCW}$ ) participants diagnosed as MDR-TB patients and registered under the DOTS Plus programme between the fourth quarter of 2013 and first quarter of 2014.

Patients who expressed willingness and given informed written consent were invited to participate in FGDs. Each FGD comprised of six to eight members both male and female. Care was taken to maximize the comfort levels of patients. FGDs generally coincided with patients drug collection day and they were offered sum of Rs. 100/- (\$1.8 at the time of the study) to meet their food and local travel expenses.

\section{Study instruments}

Aqualitative FGD guide was prepared based on the literature review and previously gained experience by the team (Box-1). The thematic areas in the guidelines included the MDR-TB patient's physical problems (pain, breathlessness, tired, dizziness, feeling sleepy), psychological disturbances (worries, fear, tension, denial, depression), social problems (disclosure issues, rejection, enacted stigma, perceived stigma, discrimination by family members, community) and economic problems (inability to go for work, work absenteeism, loss of income, borrowings). In addition, challenges they faced during treatment and further to lead normal life were covered.

\section{Data analysis}

All FGD sessions were audio recorded and the audio taped information were transcribed verbatim in Tamil and translated into English. Transcribed and translated qualitative data were entered in NVIVO software programme.

Box 1. Sample focus group discussion questions

- Have you disclosed your MDR-TB status to anyone?

- Have you had any problems with regard to disclosure of your MDR-TB status?

- What was your reaction to diagnosis of MDR-TB?

- Reactions of your family members after you were diagnosed as MDR-TB?

- Have you experienced any discrimination on account of illness?

- What do to you think are the reasons for you getting MDR-TB?

- Could you elaborate your perception \& problems you have encountered because of MDR-TB?

- Can you describe the economic problems faced due to MDR-TB illness?

- Do you have any concerns about future with MDR-TB illness?

- What might be some of the intervention strategies that would be acceptable \& feasible?

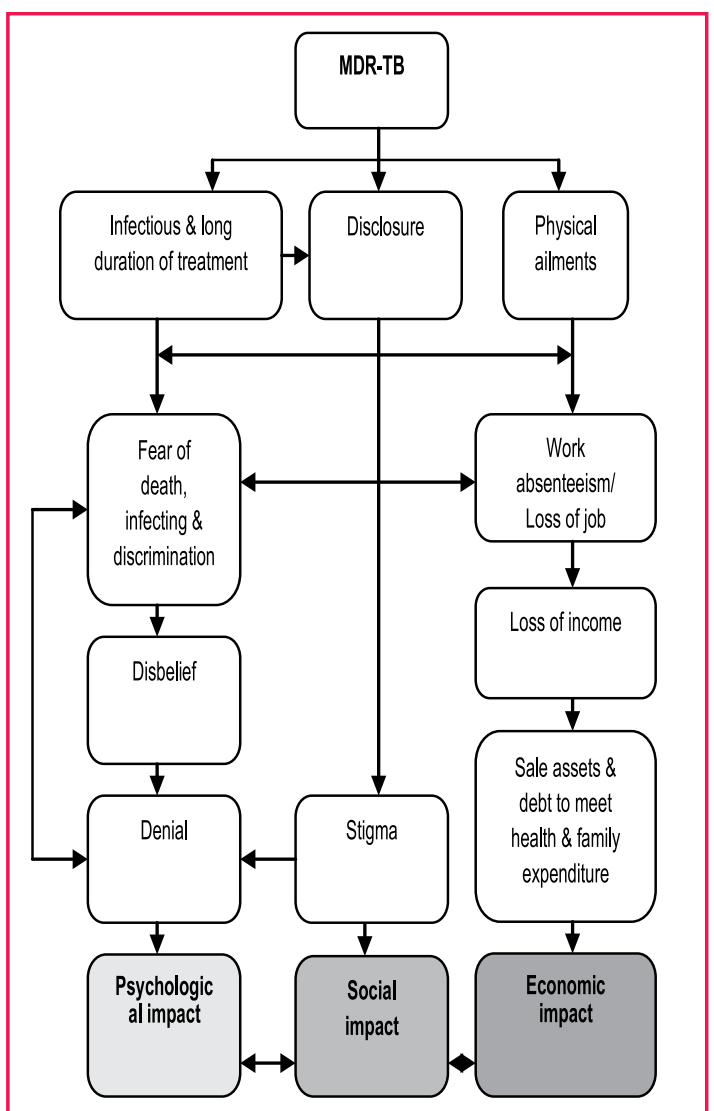

Figure 1. Conceptual framework of MDR-TB and its psychological, social and economic impact 
Content analysis and theoretical thematic interpretation has been done involving both deductive (top down) and inductive (bottom up) coding and linking codes. Based on the contextual psycho social themes which were identified in the analysis, a flow chart was developed (Figure-1). Transcribed data were divided into following five specific themes.

i) Knowledge about diagnosis of MDR-TB,

ii) Initial reaction to illness,

iii) Challenges related to disclosure issues

iv) Experience of stigma and

V) Economic problems faced by MDR-TB patients

\section{Human subject protection}

This study was approved by the Scientific Advisory Committee and Institutional Ethics Committee of NIRT, Chennai (IEC No. 2013004). Approval from Institutional Review Boards of GHTM, Tambaram, Chennai and Government Rajaji Hospital, Madurai was also obtained.

\section{RESULTS}

Profile of participants

Table 1. Socio-demographic profile of participants

\begin{tabular}{|l|l|l|}
\hline Profile & No. & $\%$ \\
\hline Gender & & \\
Male & 47 & 69 \\
Female & 21 & 31 \\
\hline Marital Status & & \\
Unmarried & 08 & 12 \\
Married & 53 & 78 \\
Widow / Widower & 03 & 04 \\
Separated & 04 & 06 \\
\hline Educational Status & & \\
Illiterate & 08 & 12 \\
Primary & 16 & 24 \\
Secondary & 34 & 50 \\
Higher Secondary & 04 & 06 \\
Degree and above & 06 & 08 \\
\hline Occupational Status & & \\
Daily Wage / Labour & 18 & 26 \\
Home maker & 10 & 15 \\
Self employed & 07 & 10 \\
Service (Pvt. \& Govt.) & 11 & 16 \\
Agriculture & 06 & 09 \\
Unemployed & 16 & 24 \\
\hline
\end{tabular}

Focus Group Discussions conducted among a sample of 68 MDR-TB patients alone was analysed and results are described in the following pages.
Study participants were aged between 19-73 years, and more than half of them were married. With respect to education majority had school level education. Except one patient, all were started treatment under Category-II regimen in RNTCP, subsequently diagnosed as MDR-TB and placed under DOTS Plus Category-IV regimen. Participants were homogenous in terms of age in three out of five FGDs. Two FGDs comprised of slightly older participants. However, participants in all five FGDs were daily wage earners.

\section{i) Knowledge about diagnosis of MDR-TB}

One third of participants did not know their MDR TB status, reasons for MDR-TB and the need for prolong duration of treatment. Participants reported that they were affected with 'muthina TB' (advanced form of TB) and 'Valarntha TB' (over grown form of TB. Few others reported that health care providers told them to receive daily injections for six months, and on medication for 24 months. However participants felt that reasons for this was not explained to them. One male participant expressed that, "I was informed that I need to take 12 tablets per day for two years with 6 months injection daily, no one informed me that I have MDR TB" (Male-age 36 years)

\section{ii) Initial reaction to illness}

One of major issues brought out by the participants was fear. Participants both men and women had undergone fear of infecting other family members, fear of death and fear over future of family members.

On hearing about long duration of treatment and getting to know quantum of drugs to be consumed the participants internalised the problem dimension and undergone series of initial reactions. More than half participants felt guilty as they were told that this illness was due to their fault of being non adherent to TB treatment. "I am not able to swallow tablets and I feel it is very hard to take 12 tablets. I vomit one or two tablets. I feel it is a heavy dosage, after taking Rx, I feel drowsy; I even get unnecessary dreams and nightmares..." (Male-age 35 years)

One college going student (male-21 years) said that his perceived stigma did not allow him to 
concentrate on his studies. He stated that "I was not OK, I cried, I could not concentrate on my exams, at this young age I got this disease"

Other major issue brought out by the participants was fear. Most of the respondents (both men and women) underwent fear of infecting other family members, fear of death and fear over future of family members. The young patients were afraid of their marriage prospects. "I am already 24 years old female, now that I have MDR-TB, will I ever get married? I am worried about my future life"

Apart from fear, other psychological issues such as disbelief, denial and depression etc., were consistently reported by every participant in the FGDs. MDR-TB has impacted sexual life also. One female participant sacrificed her family life due to fear of infecting her spouse and she said the following. "I avoid sexual contact with my husband to prevent MDR-TB infection to him" (Female-age 21 years).

"I cried. I could not concentrate on my exams. At this young age I got this disease" (Male-19 years)

The experiences of depression made participants feel self-pity over his/her disease status and other problems related to the disease. Self blaming for all that happened was a choice for many. Once this condition worsens, suicidal ideation cropped in. Disbelief and denial often led to depression and suicidal thoughts. Some of the expression of denial and disbelief by the participants were "I felt like killing myself. How was I going to take tablets for two years? ......I do not even have proper food" (Male-36 years)

"I am willing to take two years of treatment. If I am not cured this time also there is no other way and I will have to die" (Female-29 years)

\section{iii) Challenges related to disclosure issues}

Majority of the participants expressed that they did not disclose their MDR-TB status to their family members, as disclosure of TB itself was presumed as a sensitive issue, leading to rejection within the home. As one young participant (Male-age 21 years), revealed his fear of future marriage prospects. "I feared to disclose MDR-TB status to my relatives especially to my aunt owing to fear of my future" (Male-age 21 years)
Another issue of disclosure was negative impact it might generate in the neighbourhood. One of the participants (male-35 years) had expressed that his neighbours have labelled him as 'chronic sick person'. As a result he rarely moved out of his home to avoid facing uncomfortable questions from neighbours. "I am asked questions such as why I am so thin, why do I still have cough? why I am taking tablets?... I seldom left my home to avoid answering such questions" (Male, 35 years).

"I am scared to mix with any one, not attending any social gathering, not going out for any purposes and now I got used to stay alone in a separate room" (Male, 45 years).

\section{iv) Experience of stigma}

A common response among both men and women in the discussion was that MDR-TB patients faced discrimination from within and outside the home on account of prolonged treatment that required frequent visits to the hospital.

\section{a) Discrimination within family}

Most of the respondents reported that they faced discrimination within their family. This was reflected in abusive remarks either being sent out of the home or being isolated within the family. Few have opted to keep their belongings separate within the house. In many instances in-mates have neglected a MDR-TB patient altogether and considered $\mathrm{him} / \mathrm{her}$ as a burden to their family. Household discrimination in particular, makes life very difficult for MDR-TB patients. It was expressed by a male participant. "I lost my parents. My brothers and sisters are not accepting me ever since they have heard that I have TB and need to continue treatment for two years. Now I am staying on the platform" (Male-31 years).

Sometimes patient's family reacted favourably, which the patients long for. In some cases families ostracized the patient to a point of confining him/ her to the most dirty and remote part of the house and limited any kind of interaction with him/her.

Half the participants reported that their friends and neighbours ill-treated and avoided them. Participants reported experience of heartedness both directly and indirectly. Some of them also reported adverse reaction from neighbours. Two 
respondents reported that they were asked to vacate their house due to their illness. Many participants experienced discrimination in public places too. Two respondents were asked to vacate their house due to their persistent illness. Expression of two participants is narrated as "Soon after I was discharged from TB hospital, my house owner forced me to vacate the room. I was sleeping on platform" (Male-21 years)

"My neighbours keep asking me why I go to the hospital so often and make rude comments at my persistent symptoms of cough..."(Male-31 years).

This included questions as why do they (patients) make frequent visits to hospital, why they were sick "so long" etc., neighbours also made comment on their persistence cough. A male patient aged 35 years expressed self inhibition that made him stay in-door for long out of fear of discrimination. "Most of the times I never go out of my house because I could not face questions such as: why I am so thin?; why do I still have cough ?; Why I am taking tablets? ........... " (Male - 35 years).

\section{b) Discrimination by health provider}

A common response among two third of participants was the discrimination from health care provider. Participants were made to feel disgraced and discriminated at the hands of health care workers. This was highly distressing to MDR-TB patients. Delay in health care centre, attitude of health staffs to them, loud comments about their illness and not allowing proximity to them were reported. Statements expressed by the participants about health care workers are, "I am treated differently as compared to other patients attending the health care centre. I was told to move away and sarcastic comments were made." (Male - 34 years).

"When a health worker found difficult to give an injection, she scolded me rudely without any reason; because of this I was so upset and distressed....." (Female - 29 years).

"A health worker visited my house and informed my mother that I was a MDR-TB patient; further she told her that MDR-TB is dangerous and advised her not to be close to me. My relationship with my mother was ruined"' (Male29 years). v) Economic problems faced by MDR-TB patients

Often patients were the only working members of the household and most women had responsibility for running the home as well as taking care of children at home. Patients and their families were afraid of losing economic stability which was already precarious owing to disease. This fear has often, generated a great deal of stress. "I stopped going to work and stayed at home hence unable to contribute to household expenditures. My brothers and sisters used to visit me rarely and give sum of Rs. 200 to 300 and go off. I was financially affected as a result worried and felt guilty" (Male-35 years)

"I need to travel 4 to 5 kilometres daily for taking drugs. I am the bread winner of the family. I could not attend every day as I need to go for work. When I take tablets with injection I could not do any activities, I am unable to maintain regularity" (Male-31 years).

Patients from the rural areas have to travel 6-15 $\mathrm{km}$ to reach the treatment centre for drug collection and for receiving injection which costs them around Rs. 100 to 200. It was stated by a rural patient "If I visit hospital for treatment, I have to travel $15 \mathrm{~km}$ from my village. I need at least Rs. 100 per visit to spent for bus ticket for me and my wife, we also have to drink a cup of tea, If I have money we will take lunch otherwise come home with hungry" (Male-49 years).

It was presumed that urban patients have more transportation, easy access to treatment centres which made it less expensive. Interestingly it was noted though the distance to treatment centre was less; patients have to change over at least two buses or take alternate mode of transport which made them spent considerable amount for travel.

During the MDR-TB treatment, patients reported to have experienced serious and life threatening complaints including breathlessness, gastroenteritis, severe cough for which they approached private clinics or private practitioners for timely relief. Such unexpected treatment episodes incurred higher cost to the patients and even pushed them to borrow money, further making them debt. It was reported by a patient (male-49 years) who had spent Rs 2,00,000 in a 
private hospital in an urban locality over a period of six months. More than one third of the participants have expressed the need for financial or nutritional assistance during the treatment. This was narrated by a patient "I am the only earning member of the family, now I am unable go to work and cannot provide money to run the family. I also need money to go for treatment. Any financial assistance provided during treatment and nutrition will be of great help" (Male, 45 years).

\section{DISCUSSION}

This study had highlighted multiple challenges faced by MDR-TB patients such as disclosure, stigma, psychological and economic problems. These findings corroborate the findings from other studies, which have highlighted the psychological, social and economic challenges experienced by MDR-TB patients. ${ }^{7}$ This result warrants a comprehensive intervention plan to address these issues in a phased manner in order to enhance treatment adherence and better quality of life among MDR-TB patients.

One of the salient findings from this study was lack of knowledge on diagnosis of MDR-TB among patients registered under the DOTS Plus programme. From the public health perspective, knowledge on illness is an important component which helps the individual to obtain, communicate, process, and understand basic health information. This highlights the need for imparting knowledge about MDR-TB among infected patients. Participants also reported that the health care workers have not informed them about MDR-TB. This may be due the poor knowledge on MDR-TB among health care workers themselves. This was substantiated with the findings reported that only less than half of health care workers had correct knowledge on TB. ${ }^{8}$ Similarly another study among health care workers and TB patients conducted in Southern Nigeria noted that one third (38\%) of health care workers and most of the TB patients $(82 \%)$ had poor knowledge about of MDR-TB. ${ }^{9}$ It implies that there is a need for training and retraining health care workers on MDR-TB so that they can give appropriate health education for patients.

The present study found that MDR-TB patients have experienced stigma from their own family, community and health care system. The literature indicates that stigma, isolation, sense of social support, helpless feeling, and other psychological issues related to the disclosure of the diagnosis, adversely affect the TB treatment adherence. ${ }^{10,11,12,13}$ Moreover stigma also had its direct impact by affecting patients in multiple ways and it has been shown to hinder adherence to treatment. Utilizing principles of motivational interviewing which was found to be effective in improving treatment adherence in India, ${ }^{14}$ could be further explored among MDR-TB patients. In addition to educating to patients, their families and employers on MDR-TB it becomes important to help them (patients) understand their problems and help them to overcome both perceived and enacted social stigma.

It was found that both morbidity and treatment of MDR-TB produced an economically vulnerable situation for patients. Due to MDR-TB, many patients have experienced physical ailments related to their disease, such as shortness of breath, fatigue, or wasting, headaches, gastritis, depression and, peripheral neuropathy. These physical complaints diminished their ability to takeup productive work. In this study majority of the participants were from low socioeconomic status (wage labourers). They suffered an inability to work and were stressfully trying to overcome the feelings of 'uselessness' and being 'a burden' for their families. In addition TB itself limits the patient's ability to purchase food, medications and obtaining relief from other stressors. ${ }^{15}$ It was estimated by WHO that in an average drug sensitive TB patient loses three to four months of work-time and up to $30 \%$ of their household annual earnings. ${ }^{16}$ The cost per patient treated for drug-susceptible TB in 2013 was in the range of US\$100-US\$ 500 and very high for MDR-TB ranged from an average of US\$ 9235-US\$ 48553. ${ }^{17}$ All these conditions have forced MDR-TB patients to borrow money from money lenders which caused more stress, isolation and depression. One alternate strategy is to provide health insurance coverage for TB patients (for in and outpatient costs) which could critically address the TB related financial hardship. We highlight Verena et al, who emphasised the severe impact of MDR-TB related costs on the household and advocated for the inclusion of TB services in national health insurance schemes supported by food and transport subsidies. 
It was reported ${ }^{18}$ from Lima, Kazakhstan and Nepal that through psychosocial interventions treatment adherence to TB was improved. Similarly offering free treatment, hospital discounts, transportation stipends and psychosocial counselling based on the need assessment have improved treatment success. In Kazakhstan. ${ }^{19}$ 'Psychosocial support program' provided to MDR-TB patients was also successful in reducing default rates. In Nepal, ${ }^{20}$ an intervention which provided counselling support to MDR-TB patients showed that cure rate was $85 \%$.

\section{Suggested psychosocial intervention strategies}

These findings highlight the need to apply an integrated intervention model that includes counselling and active health education in addition to incorporating successful experiences of generic models of service delivery. In this direction, the researchers have compiled and regrouped the findings under three broad areas viz., psychological challenge, socio-economic challenge, and medication challenge. As a result the researchers suggest an intervention model (please refer to Figure 2) that would alleviate sufferings experienced by MDR TB patients.

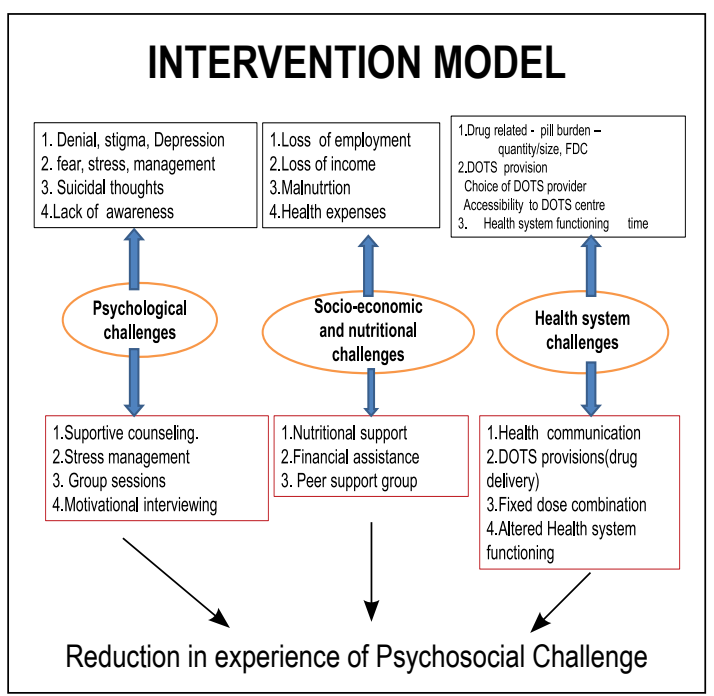

Figure 2. Intervention Model to cope psycho social challenges faced by MDR-TB.

- Psychological support can be offered by either Medical social worker/counsellor or psychiatrist. One to one individual counselling session with psychologist or medical social worker could help in clarifying many of their doubts/fears and reducing their psycho social sufferings. Health education should be offered before initiating treatment as observed in this study.

- Socio-economic support in terms of monetary assistance, nutritional support can be offered to mitigate financial hardship experienced as a result of illness.

- Wherever feasible peer involvement can be promoted, peer participation strategies which was found successful in HIV prevention.

- Medication support can be explored from medicine to DOTSs provision. DOTS can be further simplified and arranged near to patient's residence. Reduction in number of tablets perhaps fixed dose combination, change in health system (DOTS centre) functioning can also be explored.

\section{Limitations of the study}

This study was conducted among MDR-TB patients registered under DOTS Plus programme in government health facilities. Results produced from this research may be unique to the study setting in which we have conducted and may not be readily generalized to other settings. Furthermore we used qualitative tool to collect information for the purpose of this study which has its own limitations. However, our work highlights unexplored issues that are likely to be relevant to many developing countries with high MDR-TB burden, particularly those with a similar socioeconomic status.

\section{CONCLUSION}

This qualitative study has revealed significant psychological, social, and financial challenges associated with MDR-TB to patients and their families. There is a need for psychosocial support of MDR-TB patient and their caregivers to mitigate the negative effects of stigma, and to manage the associated psychological stressors. Our findings also raise several policy relevant issues in the management of MDR-TB in the community. There should be policy debates and reforms to innovate intervention strategies, which could aid the MDR-TB patients to cope with their psychosocial challenges leading to improved treatment adherence, reduced default and TB transmission rates. Besides this there is a critical need to examine the patients' financial concerns about higher treatment cost, transportation cost and loss of income. 


\section{REFERENCES}

1. World health Organisation Global Tuberculosis Report 2014. http://www.who.int/tb/publications/ global_report/gtbr14_main_text.pdf

2. World health Organisation. Multi Drug Resistant Tuberculosis(MDR-TB). World Health Organisation 2014. http://www.who.int/tb/challenges/mdr/

3. Central TB Division. RNTCP Response to Challenges of Drug resistant TB in India. Central TB Division, Directorate General of Health Services, Ministry of Health \& Family Welfare, Government of India, New Delhi 2012. http://www.tbcindia.nic. in/pdfs

4. Institute of Medicine (US). Facing the Reality of Drug-Resistant Tuberculosis in India: Challenges and Potential Solutions: Summary of a Joint Workshop by the Institute of Medicine, the Indian National Science Academy, and the Indian Council of Medical Research. Washington (DC): National Academies Press (US); 2012.Available from: http:// www.ncbi.nIm.nih.gov/books / NBK92617/

5. World Health Organisation. The Global Plan to STOP TB 2011-2015, WHO, Geneva, 2010; www. stoptb.org/global/plan

6. Central TB Division. Revised National Tuberculosis Control Programme: DOTS-Plus Guidelines. Central TB Division, Directorate General of Health Services, Ministry of Health \& Family Welfare, Government of India, New Delhi 2010.

7. Meghan D. Morris, Liliana Quezada, Priya Bhat, Kathleen Moser, Jennifer Smith, et al. Social, Economic, and Psychological Impacts of MDRTB Treatment in Tijuana, Mexico: A Patient's Perspective. Int J Tuberc Lung Dis 2013;17(7):954960

8. Malangu N, Adebanjo OD. Knowledge and practices about multidrug-resistant tuberculosis amongst healthcare workers in Maseru. Afr J Prm Health Care Fam Med. 2015;7(1):774-779.

9. Alphonsus RI, Alexander A, et al. Concerns about the knowledge of multi drug resistant tuberculosis among health care workers and patients in Southern Nigeria. Am J Respir Crit Care Med 2014;189:A3215

10. Kelly P. Isolation and stigma: the experience of patients with active tuberculosis. Journal of Community Health Nursing 1999;16(10):233-41
11. Munro SA, Lewin SA, Smith HJ, Engel ME, Fretheim A, Volmink J. Patient adherence to tuberculosis treatment: a systematic review of qualitative research. PLoS Medicine 2007;4(7):1230-45

12. Naidoo p, K. Mwaba, "Helplessness, depression, and social support among people being treated for tuberculosis in South Africa," Social Behavior and Personality, vol. 38, no. 2010;10, pp.1323-34

13. G. D. Natani, N. K. Jain, and T. N. Sharma. Depression in tuberculosis patients: correlation with duration of disease and response to antituberculous chemotherapy, Indian Journal of Tuberculosis, vol. 32, 1985;4,pp.195-98

14. Janmeja, A.K., Das, S.K., Bhargava, R. and Chavan, B.S. Psychotherapy Improves compliance with tuberculosis treatment. Respiration, $2005 ; 72,375-80$

15. Kelly MP, Bonnefoy J, Morgan A, Florenza A. The development of the evidence base about the social determinants of health. World Health Organization Commission on Social Determinants of HealthMeasurement and Evidence Knowledge Network. 2006;http://www.who.int/social_determinants/ resources/mekn_paper.pdf

16. Global Alliance for TB Drug Development. Economic impact of TB. http://www.tballiance.org/ why/economic-impact.php

17. World Health Organization. Global Tuberculosis Report 2014. WHO, Geneva 2014. http:// www.who.int/ tb/ publications/ global report/ gtbr14executivesummary.pdf

18. Sonya Shin, Jennifer Furin, Jaime Bayona, Kedar Mate, Jim YongKim, Paul Farmer. Communitybased treatment of multidrug-resistant tuberculosis in Lima, Peru: 7 years of experience. Social Science \& Medicine 2004;59:1529-39.

19. Kaliakbarova G, PakS, Zhaksylykova N, Raimova G, Temerbekova B, van den Hof S. Psychosocial Support Improves Treatment Adherence Among MDR-TB Patients: Experience from East Kazakhstan. The Open Infectious Diseases Journal 2013;7:(Suppl 1: M7)60-64.

20. Sushil C Baral, Yeshoda Aryal, Rekha Bhattrai, Rebecca King, James N Newell. The importance of providing counseling and financial support to patients receiving treatment for multi-drug resistant TB: mixed method qualitative and pilot intervention studies. BMC Public Health 2014;14:46(1-7) 